\title{
Terminal QRS Distortion on Admission Electrocardiogram as a Predictor of Left Ventricular Systolic Dysfunction in Patients with ST Elevation Myocardial Infarction
}

\author{
Mohammad Morshedul Ahsan, Md. Abdul Kader Akanda, Sabina Hashem, Md. Zillur Rahman \\ Mohammad Khalilur Rahman Siddiqui, Surovi Sultana, Abeeda Tasmin Reza, Ratan Kumar Datta, \\ Khaled Mahmood, Pranob Karmaker \\ Department of Cardiology, National Institute of Cardiovascular Diseases, Dhaka
}

Key words:
Terminal QRS
distortion, Left
ventricular
systolic
dysfunction, ST
elevation
myocardial
infarction.

Abstract:

Background: Increased mortality rates due to coronary artery disease remains a big concern inthe field of cardiology. So early risk stratification is important in daily clinical practice. Risk assessment needs to be done shortly after admission, when only the history, physical examination and the ECG are available. The role of ECG in diagnosis and prognostication of acute myocardial infarction is well established. So this study was done to find out the association of terminal QRS distortion with left ventricular systolic dysfunction in patients with acute ST Elevation Myocardial Infarction.

Methods: This prospective observational study was done in NICVD with acute STEMI patients who were eligible for thrombolysis, and was admitted in coronary care unit within 12 hours of onset of chest pain. After considering inclusion and exclusion criteria total 200 acute STEMI patients were taken purposively. 100 patients in group I with terminal QRS distortion and 100 patients in group II without terminal QRS distortion. Then Echocardiography was done to assess left ventricular systolic dysfunction.

Results: In our study mean ejection fraction was significantly lower in group I (42.6 $\pm 5.4 \%$ vs $49.7 \pm 5.3 \%$, $p=0.001$ ). Mean ejection fraction was $40.4 \pm 4.7 \%$ in terminal $Q R S$ distortion patients with anterior $M I$ and $47.9 \pm 5.9 \%$ in patients without terminal $Q R S$ distortion with anterior $M I(p=0.001)$. Mean ejection fraction was $46.6 \pm 6.4 \%$ in terminal $Q R S$ distortion patients with inferior MI and $53.9 \pm 7.8 \%$ in patients without terminal QRS distortion patients with inferior MI ( $p=0.003)$. Out of the 7 variables location of MI and terminal QRS distortion were found to be the independent predictors for developing left ventricular systolic dysfunction with ORs being 5.20 and 3.21.respectively.

Conclusion: ECG on admission in STEMI can predict short term prognosis and help in early assessment of severity of ischemia. Presence of terminal QRS distortion on admission ECG in STEMI is associated with LV systolic dysfunction. So it helps in early decision making for referral to a higher center by our grass root level doctors.

(Cardiovasc. j. 2018; 11(1): 42-49)

\section{Introduction:}

A recent study from rural Bangladesh demonstrated a dramatic increase in CVD from 1986 to $2006 .{ }^{1}$ In this scenario of increased mortality rates due to coronary artery disease early risk stratification is important in daily clinical practice to predict the infarct size, success of epicardial recanalization and risk of adverse outcomes in patients presenting with ST-segment elevation myocardial infarction (STEMI). The 12 leads electrocardiogram (ECG) is the most readily available noninvasive test by which in addition to diagnosis, we can also localize and assume the size of myocardial infarction.

ST segment elevation is used to define subsets of patients who can benefit most from early thrombolysis. ${ }^{2}$ A specific QRS morphology termed as terminal QRS distortion is independently associated with increased in hospital mortality. ${ }^{3}$ Patients experiencing their first anterior AMI who have terminal QRS distortion on the admission ECG have more

Address of Correspondence: Dr.Mohammad Morshedul Ahsan, Department of Cardiology, National Institute of Cardiovascular Diseases, Dhaka, Bangladesh. Email: morshedul_ahsan@yahoo.com 
severely depressed LV wall motion and less improvement in regional wall motion in the infarct region during the healing stage, suggesting that it may be an indicator of severe and irreversible ischemic damage. ${ }^{4}$ And it is better than ST-segment measurements in predicting final Infarct size and assessing the potential effect of thrombolytic therapy in anterior wall acute myocardial Infarction. ${ }^{5}$

Terminal QRS distortion is characterized by emergence of $\mathrm{j}$ point $\mathrm{e}$ " $50 \%$ of the $\mathrm{R}$ wave in leads with $\mathrm{qR}$ configuration and/or disappearance of the $\mathrm{S}$ wave in leads with $\mathrm{Rs}$ configuration. The changes in the terminal portion of the QRS are believed to be caused by prolongation of the electrical conduction in either the Purkinje fibers or the myocardium in the ischemic zone and reflect severe ischemia. ${ }^{6}$ The severity of ischemia /infarction process is determined by the degree of myocardial protection provided by the combination of collateral vessels and ischemic preconditioning .For practical purposes, severity of ischemia is categorized into three grades; grade 1: tall symmetrical $\mathrm{T}$ waves without STE; grade 2: STE without terminal QRS distortion; and grade 3: STE with terminal QRS distortion. ${ }^{7}$

Birnbaum hypothesized that absence of terminal QRS distortion on the ECG of patients presenting with acute STE myocardial infarction (STEMI) is a sign of myocardial protection by persistent perfusion (via collateral circulation or incomplete or intermittent epicardial artery occlusion) or preconditioning via ischemia or medications. ${ }^{7}$ The patients with terminal QRS distortion group shows larger area at risk (AAR) , larger infarct size and worse left ventricular ejection fraction. ${ }^{8}$

In leads with usual Rs configuration (leads V1V3): grade I,tall symmetrical T wave without ST elevation;grade II, ST elevation without distortion of theterminal portion of the QRS complex; grade III, ST elevation with distortion of the terminal portionof the QRS (no $\mathrm{S}$ waves in leads V1-V3) (arrow).

In leads with usual qR configuration: grade I,tall symmetrical $\mathrm{T}$ wave without ST elevation; grade II, ST elevation with $J$ point/R wave ratio $<0.5$; grade III, ST elevation with $\mathrm{J}$ point/R wave ratio $>0.5$ (arrow). ${ }^{9}$

Line a: QR complexes without terminal QRS distortion. Despite different degree of STsegment

elevation, in all complexes the $\mathrm{J}$ point (arrow) emerges below $50 \%$ of the $\mathrm{R}$-wave amplitude (dashed line). Line b: QR complexes with terminal QRS distortion. In all complexes, the J point emerges above $50 \%$ of the $\mathrm{R}$-wave amplitude. Line c: RS complexes without terminal QRS distortion. In all complexes the $\mathrm{S}$ wave is preserved (compared with line d). Line $\mathrm{d}$ : complexes in leads usually demonstrating RS pattern with terminal QRS distortion showing different magnitude of ST segment elevation. However, in all complexes $\mathrm{S}$ wave disappeared.

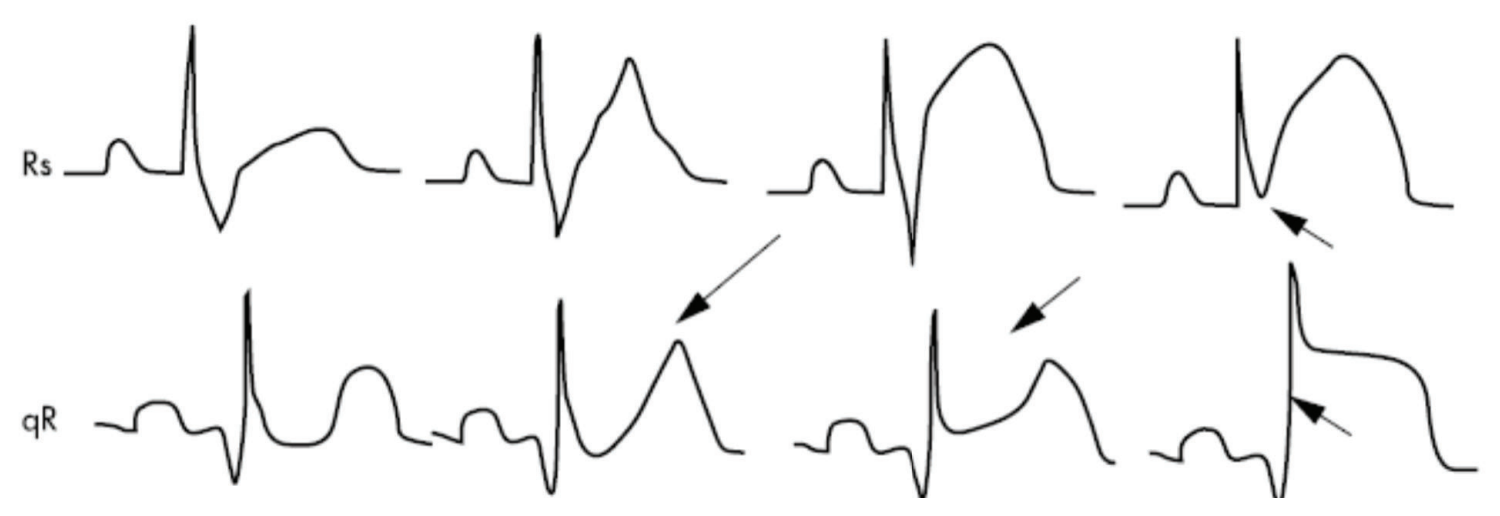

Fig.-1: The grades of ischaemia. 


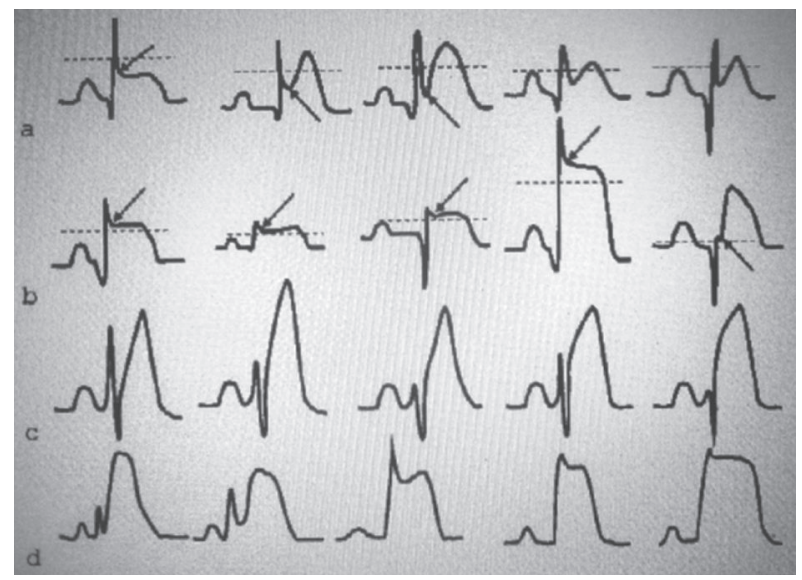

Fig.-2: Terminal QRS distortion ${ }^{3}$

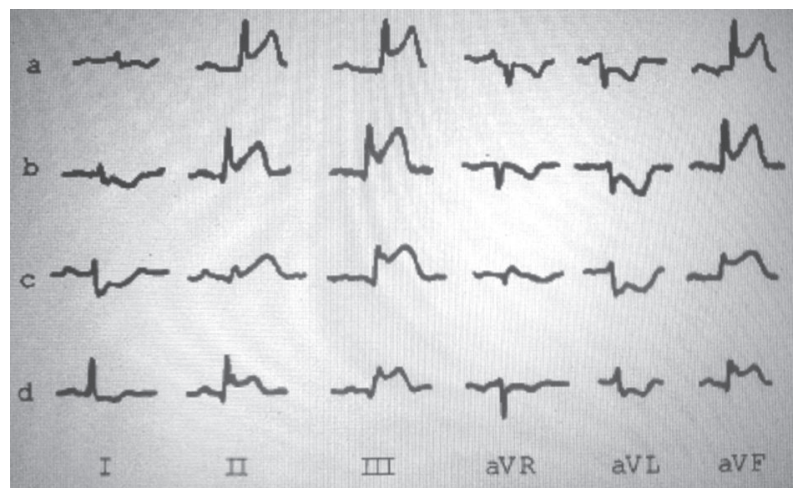

Fig.-3: Terminal QRS distortion in inferior $M I$. Examples $a$ and $b$ are STEMI without.

Terminal QRS distortion as the J points in lead II ,III, AVF are less than $50 \%$ of the $\mathrm{R}$ wave height. Example c and d are STEMI with Terminal QRS distortion as the J points in lead III and AVF are more than $50 \%$ of the $\mathrm{R}$ wave height. ${ }^{3}$

\section{Methods:}

This prospective observational study was conducted at the Department of Cardiology, National Institute of Cardiovascular Diseases and Hospital, Dhaka, during the period from May 2015 to October 2016.By purposive sampling technique 200 cases were taken. Informed written consent was taken from each patient before enrollment. Acute STEMI patients who were eligible for thrombolysis, and were admitted in coronary care unit within 12 hours of onset of chest pain were considered for the study after considering inclusion and exclusion criteria. Meticulous history was taken and detailed clinical examination was performed and recorded in predesigned structured proforma. Patient's data including age, sex, previous history of anginal chest pain, smoking, hypertension (HTN), diabetes mellitus (DM), family history of CAD and clinical findings on admission were taken. Results of routine investigations such as RBS, serum Creatinine, serum electrolytes, Troponin-I, were noted. ECG: 12 lead resting ECG were done at a paper speed of $25 \mathrm{~mm} / \mathrm{s}$ and $10 \mathrm{~mm}$ standardization immediately after admission by using Zoncare single channel ECG machine. STEMI was diagnosed when ECG showed ST elevation of $0.1 \mathrm{mv}$ in two or more limb leads or $0.2 \mathrm{mv}$ in two or more consecutive chest leads with increase in cardiac enzymes. Anterior MI was considered when ST elevation of at least $0.2 \mathrm{mv}$ was present in any two or more consecutive leads from V1 to V6. And Inferior MI was considered when ST elevation of at least $0.1 \mathrm{mv}$ was present in any two of Lead II, III and aVF.

ECG were analyzed for Presence of terminal QRS distortion. Terminal QRS distortion is characterized by emergence of $\mathrm{j}$ point $\mathrm{e}$ " $50 \%$ of the $\mathrm{R}$ wave in leads with $\mathrm{qR}$ configuration and/ or disappearance of the $\mathrm{S}$ wave in leads with $\mathrm{Rs}$ configuration. The cases were divided into two groups, 100 consecutive patients with terminal QRS distortion were taken in group I and 100 patients without terminal QRS distortion matched with site of infarction were taken in group II. Group I again subdivided into anterior MI and inferior MI, each having 50 patients. And group II again subdivided into anterior $\mathrm{MI}$ and inferior MI each having 50 patients. ECG was analyzed by three experienced physicians who were blinded to clinical data of the patients. Continuous ECG monitoring was done for first 24 hours in CCU and 12 lead ECG were repeated every 24 hours and when necessary. Clinical assessment and various risk factors were noted. All patients had received streptokinase 1.5 million units infusion over a period of $1 \mathrm{hr}$. and standard medications like beta blockers, ACEI/ ARBS, statins and antiplatelets as per the discretion of the treating physician. Echocardiography was done by SIEMENS ACUSON X 700 echocardiography machine. Results of 2D, M-mode transthoracic 
echocardiography such as left ventricular internal diameter in diastole (LVIDd), left ventricular internal diameter in systole (LVIDs) and left ventricular ejection fraction (LVEF) was noted on day two. LVEF was measured by modified Simpson method. Patients were monitored at CCU for at least 24 hours and then at ward till discharge or death. Patients were followed up regularly for monitoring in hospital outcome. Following variables were studied: Age, Sex, Smoking, HTN, DM, Family history of premature $\mathrm{CAD}$, left ventricular ejection fraction (LVEF)

The numerical data obtained from the study were analyzed and significance of differences were estimated by using statistical methods. The Statistical Package for Social Sciences version 16 software (SPSS Inc., Chicago, Illinois, USA) was used for data analysis. Qualitative data like sex, smoking, hypertension, Diabetes mellitus, family history of premature coronary artery disease, chest pain, shortness of breath, vomiting, sweating, syncope, palpitation, recurrent ischemia, heart failure, significant arrhythmia, cardiogenic shock and death were expressed as percentage and frequency and difference between two groups were analyzed by Chi -square test and Fisher's exact test. Quantitative data like age, pulse, blood pressure, RBS, serum creatinine, serum electrolytes, LVEF, and hospital stay were expressed as mean and standard deviation. And difference between two groups was analyzed by the Student's t-test. Multivariate logistic regression analysis was done to exclude confounding variables for LV systolic dysfunction. Odds ratio was analyzed to assess the risk of imposed QRS distortion for left ventricular systolic dysfunction. A p- value of less than 0.05 will be considered statistically significant.

\section{Results:}

The mean age was found $55.31 \pm 11.81$ years with QRS distortion group and $52.60 \pm 10.45$ years without QRS distortion group. Analysis revealed statistically not significant mean age difference $(p=0.08)$ between two groups in unpaired t-test. In our study 160 (80\%) patients were male and $40(20 \%)$ patients were female. Male and female ratio was $4: 1$. In group I, $78(78 \%)$ patients were male and $22(22 \%)$ patients were female. In group II, $82(82 \%)$ patients were male and $18(18 \%)$ patients were female. There is no statistical significance $(p=0.48)$ in the sex distribution of the study population by Chi Square test.

Patients with terminal QRS distortion group had highest percentage of smoking (73\%), followed by diabetes mellitus (45\%), hypertension (43\%), and family history of premature coronary artery disease (22\%). Similarly, patients without terminal QRS distortion group, smoking, hypertension and diabetes mellitus were predominant $71 \%, 40 \%$ and $38 \%$, followed by family history of coronary artery disease (15\%).

Table-I

Distribution of the study population according to age and sex $(N=200)$.

\begin{tabular}{lcccccc}
\hline Age in years & \multicolumn{2}{c}{ Group I(n = 100) } & & \multicolumn{2}{c}{ Group II $(\mathrm{n}=100)$} & p value \\
\cline { 2 - 3 } & Number & $\%$ & & Number & $\%$ & \\
\hline $25-35$ & 6 & 6.0 & & 5 & 5.0 & \\
$36-45$ & 18 & 18.0 & & 22 & 22.0 & \\
$46-55$ & 27 & 27.0 & & 35 & 35.0 & \\
$56-65$ & 32 & 32.0 & & 31 & 31.0 & $0.08^{\text {ns }}$ \\
$>65$ & 17 & 17.0 & & 7 & 7.0 & $0.48^{\text {ns }}$ \\
Mean \pm SD & \multicolumn{2}{c}{$55.31 \pm 11.81$} & & \multicolumn{2}{c}{$52.60 \pm 10.45$} & \\
Sex & 78 & 78 & & 22 & 22 & \\
male & 82 & 82 & & 18 & 18 & \\
Female & & &
\end{tabular}

$\mathrm{p}$ value reached from unpaired t-test.\&Chi Square test. ns- Not significant.

Group I = patients with terminal QRS distortion

Group II= patients without terminal QRS distortion 
Table-II

Distribution of the study population according to cardiovascular risk factors $(N=200)$.

\begin{tabular}{lccccc}
\hline Risk Factors & \multicolumn{2}{c}{ Group I $(\mathrm{n}=100)$} & \multicolumn{2}{c}{ Group II $(\mathrm{n}=100)$} & $\mathrm{p}$ value \\
& Number & $\%$ & Number & $\%$ & \\
\hline Smoking & 73 & 73.0 & 71 & 71.0 & $0.53^{\mathrm{ns}}$ \\
Hypertension & 43 & 43.0 & 40 & 40.0 & $1.66^{\mathrm{ns}}$ \\
$\begin{array}{l}\text { Diabetes mellitus } \\
\text { Family history of }\end{array}$ & 45 & 45.0 & 38 & 38.0 & $0.31^{\mathrm{ns}}$ \\
premature CAD & 22 & 22.0 & 15 & 15.0 & $0.20^{\mathrm{ns}}$ \\
\hline
\end{tabular}

Data were analyzed using Chi Square test. ns- Not significant.

Group I = patients with terminal QRS distortion

Group II= patients without terminal QRS distortion

All of the risk factors were found no statistical significant difference between the two groups $(\mathrm{p}>0.05)$.

All the patients were evaluated with echocardiography to see the LV ejection fraction. Mean ejection fraction was $42.6 \pm 5.4 \%$ in group I and $49.7 \pm 5.3 \%$ in group II. Statistically significant difference in ejection fraction was found between the two group of patients $(p=0.001)$. So terminal QRS distortion is associated with lower ejection fraction and increased left ventricular systolic dysfunction.

All the patients were evaluated with echocardiography to see the LV ejection fraction. Mean ejection fraction was $40.4 \pm 4.7 \%$ in terminal QRS distortion patients with anterior MI and $47.9 \pm 5.9 \%$ in patients without terminal QRS distortion with anterior MI. It indicates that LVEF was significantly lower in terminal QRS distortion patients with anterior MI than without terminal QRS distortion patients with anterior MI $(\mathrm{p}=0.001)$.

All the patients were evaluated with echocardiography to see the LV ejection fraction.
Mean ejection fraction was $46.6 \pm 6.4 \%$ in terminal QRS distortion patients with inferior MI and $53.9 \pm 7.8 \%$ in patients without terminal QRS distortion patients with inferior MI. It indicates that LVEF was significantly lower in terminal QRS distortion patients with inferior MI than without terminal QRS distortion patients with inferior MI $(p=0.003)$.

All the patients were evaluated with echocardiography to see the LV ejection fraction. Mean ejection fraction was $40.4 \pm 4.7$ in patients with terminal QRS distortion in anterior MI. and

$46.6 \pm 6.4 \%$ in terminal QRS distortion patients with inferior MI. It indicates that LVEF was significantly lower in terminal QRS distortion patients with anterior MI than terminal QRS distortion patients with inferior MI $(p=0.004)$.

Binary logistic regression analysis of Odds Ratio for characteristics of the subjects likely to develop left ventricular systolic dysfunction was done. Taking LVEF $<45 \%$ as moderate LV systolic dysfunction this analysis was done. The variables revealed to be significantly associated with left ventricular systolic dysfunction by multivariate

Table-III

Comparison of Left ventricular ejection fraction (LVEF) between two groups ( $N=200)$.

\begin{tabular}{lcccccc}
\hline LVEF in \% & \multicolumn{2}{c}{ Group I $(\mathrm{n}=100)$} & & \multicolumn{2}{c}{ Group II $(\mathrm{n}=100)$} & $\mathrm{p}$ value \\
\cline { 2 - 3 } & Number & $\%$ & & Number & $\%$ & \\
\hline S35 (Severe Dysfunction) & 10 & 10.0 & & 03 & 3.0 & $0.04^{\mathrm{s}}$ \\
$36-44$ (Moderate dysfunction) & 50 & 50.0 & & 12 & 12.0 & $<0.001^{\mathrm{s}}$ \\
$45-54$ (Mild dysfunction) & 31 & 31.0 & & 63 & 63.0 & $<0.001^{\mathrm{s}}$ \\
$\geq 55$ (Normal LV function) & 9 & 9.0 & & 22 & 22.0 & $0.01^{\mathrm{s}}$ \\
Mean \pm SD & \multicolumn{2}{c}{$42.6 \pm 5.4$} & & & $49.7 \pm 5.3$ & $0.001^{\mathrm{s}}$ \\
\hline
\end{tabular}

P value reached from Chi-square test, Fisher's Exact test and unpaired t-test.

$\mathrm{s}$-significant. $\mathrm{ns}=$ not significant

Group I = patients with terminal QRS distortion

Group II= patients without terminal QRS distortion 
analysis were all entered into the model directly. Out of the 7 variables anterior MI and terminal QRS distortion were found to be the independent predictors for developing left ventricular systolic dysfunction with ORs being 5.20 and 3.21 respectively. Rest of the variables were not observed as independent predictors for developing left ventricular systolic dysfunction $(\mathrm{p}>0.05)$.

\section{Table-IV}

Comparison of Left ventricular ejection fraction (LVEF) between Group I anterior with Group II anterior. $(N=100)$.

\begin{tabular}{|c|c|c|c|c|c|}
\hline \multirow[t]{2}{*}{ LVEF (\%) } & \multicolumn{2}{|c|}{$\begin{array}{l}\text { Group I (Anterior) } \\
(\mathrm{n}=50)\end{array}$} & \multicolumn{2}{|c|}{$\begin{array}{c}\text { Group II (Anterior) } \\
(\mathrm{n}=50)\end{array}$} & \multirow[t]{2}{*}{$\mathrm{p}$ value } \\
\hline & Number & $\%$ & Number & $\%$ & \\
\hline$\leq 35$ (Severe dysfunction) & 8 & 16.0 & 2 & 4.0 & $0.04^{\mathrm{s}}$ \\
\hline $36-44$ (Moderate dysfunction) & 32 & 64.0 & 8 & 16.0 & $<0.001^{\mathrm{s}}$ \\
\hline $45-54$ (Mild dysfunction) & 8 & 16.0 & 35 & 70.0 & $<0.001^{\mathrm{s}}$ \\
\hline$\geq 55$ (Normal LV function) & 2 & 4.0 & 5 & 10.0 & $0.23^{\mathrm{ns}}$ \\
\hline Mean $\pm \mathrm{SD}$ & \multicolumn{2}{|c|}{$40.4 \pm 4.7$} & \multicolumn{2}{|c|}{$47.9 \pm 5.9$} & $0.001^{\mathrm{s}}$ \\
\hline
\end{tabular}

P value reached from Chi-square test, Fisher's Exact test and unpaired t-test. s-significant. ns= not significant

Group I = patients with terminal QRS distortion

Group II= patients without terminal QRS distortion

Table-V

Comparison of Left ventricular ejection fraction (LVEF) between Group I inferior with Group II inferior. $(N=100)$.

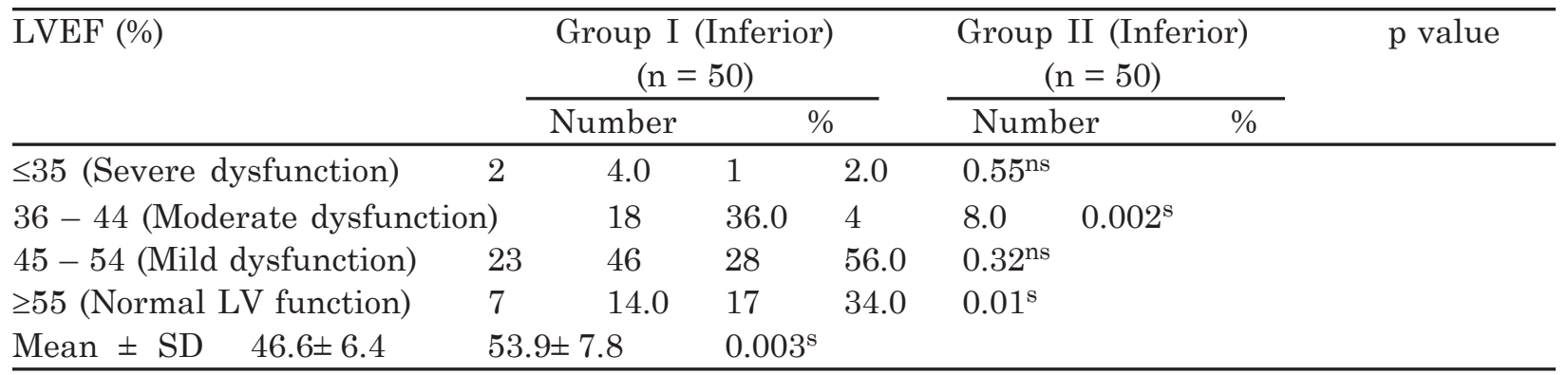

P value reached from Chi-square test, Fisher's Exact test and unpaired t-test.s-significant. ns= not significant

Group I = patients with terminal QRS distortion

Group II= patients without terminal QRS distortion

\section{Table-VI}

Comparison of Left ventricular ejection fraction (LVEF) between Group I anterior with Group I inferior. $(N=100)$

\begin{tabular}{|c|c|c|c|c|c|}
\hline \multirow[t]{2}{*}{$\overline{\mathrm{LVEF}(\%)}$} & \multicolumn{2}{|c|}{$\begin{array}{c}\text { Group I (Anterior) } \\
(\mathrm{n}=50)\end{array}$} & \multicolumn{2}{|c|}{$\begin{array}{c}\text { Group II (Inferior) } \\
(\mathrm{n}=50)\end{array}$} & \multirow[t]{2}{*}{$\mathrm{p}$ value } \\
\hline & Number & $\%$ & Number & $\%$ & \\
\hline$\leq 35$ (Severe dysfunction) & 8 & 16.0 & 2 & 4.0 & $0.04^{\mathrm{s}}$ \\
\hline 36 - 44 (Moderate dysfunction) & 32 & 64.0 & 18 & 36.0 & $0.005^{\mathrm{s}}$ \\
\hline $45-54$ (Mild dysfunction) & 8 & 16.0 & 23 & 46.0 & $0.001^{\mathrm{s}}$ \\
\hline$\geq 55$ (Normal LV function) & 2 & 4.0 & 7 & 14.0 & $0.06^{\text {ns }}$ \\
\hline Mean $\pm \mathrm{SD}$ & \multicolumn{2}{|c|}{$40.4 \pm 4.7$} & \multicolumn{2}{|c|}{$46.6 \pm 6.4$} & $0.004^{\mathrm{s}}$ \\
\hline
\end{tabular}

P value reached from Chi-square test, Fisher's Exact test and unpaired t-test.s-significant. ns= not significant

Group I = patients with terminal QRS distortion 
Table-VII

Factors related to left ventricular systolic dysfunction as dependent variable.

\begin{tabular}{lccc}
\hline Variables of interest & \multicolumn{3}{c}{ Multivariate analysis } \\
\cline { 2 - 4 } & OR & $95 \%$ CI of OR & p value \\
\hline age $(\geq 60)$ & 1.00 & $0.294-1.466$ & $0.75^{\mathrm{ns}}$ \\
Male sex & 1.23 & $0.656-3.157$ & $0.23^{\mathrm{ns}}$ \\
Smoking & 1.57 & $0.624-3.965$ & $0.33^{\mathrm{ns}}$ \\
Diabetes mellitus & 2.31 & $0.955-5.842$ & $0.06^{\mathrm{ns}}$ \\
Hypertension & 1.17 & $0.588-2.343$ & $0.54^{\mathrm{ns}}$ \\
Anterior MI & 5.20 & $1.211-10.261$ & $0.02^{\mathrm{s}}$ \\
Terminal QRS distortion & 3.21 & $2.101-5.752$ & $0.02^{\mathrm{s}}$ \\
\hline
\end{tabular}

$\mathrm{s}=$ Significant, $\mathrm{ns}=$ Not significant

\section{Discussion:}

The age of the study populations ranges from 31 to 75 years. The mean age was found $55.31 \pm 11.81$ years with QRS distortion group and $52.60 \pm 10.45$ years without QRS distortion group. In BRAVE study, Chowdhury ${ }^{10}$ found mean age of MI cases $53 \pm 10$ years. So our finding is consistent with their findings.

Among 200 patients 160 (80\%) patients of the study were male and $40(20 \%)$ patients were female. Male and female ratio was 4:1.In almost all studies related to coronary artery disease (CAD) similar male preponderance was found. As females are given less attention and access for them to the health care facilities is limited particularly in low socioeconomic population like our country may contribute for this male predominance.

Patients with terminal QRS distortion group had highest percentage of smoking (73\%), followed by diabetes mellitus (45\%), hypertension (43\%) and family history of premature coronary artery disease (22\%). Similarly, patients without terminal QRS distortion group, smoking, hypertension and diabetes mellitus were predominant $71 \%, 40 \%$ and $38 \%$, followed by family history of coronary artery disease (15\%). Akanda ${ }^{11}$ found smoking as the most prevalent $(60 \%)$ risk factor among the patients of coronary artery disease of the Bangladeshi population.

All the patients were evaluated with echocardiography to see the LV ejection fraction. Mean ejection fraction was $42.6 \pm 5.4 \%$ in group I and $49.7 \pm 5.3 \%$ in group II. Statistically significant difference in ejection fraction was found between the two group of patients $(p=0.001)$. Mean ejection fraction was $40.4 \pm 4.7 \%$ in terminal QRS distortion patients with anterior $\mathrm{MI}$ and $47.9 \pm 5.9 \%$ in patients without terminal QRS distortion with anterior MI.

Fernandez ${ }^{8}$ found worse LVEF $(41.5 \%$ vs $46.3 \%$, $\mathrm{p}=0.004$ ), in acute STEMI anterior in patients with or without terminal QRS distortion respectively.Yalcinkaya ${ }^{12}$ found Left ventricular ejection fraction $(44.58 \pm 7.23 \%$ vs $48.44 \pm 7.61 \%$ $\mathrm{p}=0.001$ ) in study population with or without terminal QRS distortion group. So our study finding is consistent with their finding. So we can assume that terminal QRS distortion is associated with increased LV systolic dysfunction.

The binary logistic regression analysis of Odds Ratio for characteristics of the subjects likely to develop left ventricular systolic dysfunction we found that location of MI and terminal QRS distortion were the independent predictors for developing left ventricular systolic dysfunction. So from above discussion we can conclude that terminal QRS distortion on admission electrocardiogram is associated with left ventricular systolic dysfunction and adverse in hospital outcome in patients with acute STEMI.

\section{Conclusion}

It may be concluded that terminal QRS distortion on admission electrocardiogram is associated left ventricular systolic dysfunction in patients with acute ST elevation myocardial infarction.

Conflict of Interest - None. 


\section{References:}

1. Karar AZ, Alam N, Streatfield KP. Epidemiological transition in rural Bangladesh, 1986-2006. Global Health Action2009; 2: 1-24.

2. Mulay DV, Mukhedkar SM. Prognostic significance of the distortion of terminal portion of QRS complex on admission electrocardiogram in ST segment elevation myocardial infarction. Indian Heart Journal 2013; 65: 671-677.

3. Birnbaum Y, Kloner RA, Sclarovsky S. Distortion of the Terminal Portion of the QRS on the Admission Electrocardiogram in Acute Myocardial Infarction and Correlation with Infarct Size and Long-Term Prognosis (Thrombolysis in Myocardial Infarction 4 Tria1). Am J Cardiol 1996; 78: 396-402.

4. TamuraA, Kimiaki N, Toru W, Masaru N. Relationship between Terminal QRS Distortion on the Admission Electrocardiogram and the Time Course of Left Ventricular Wall Motion in Anterior Wall Acute Myocardial Infarction. Japanese Circulation Journal 2001;65: 63-66.

5. Birnbaum Y, Maynard C, Wolfe S, Strasberg B, Mager A. Terminal QRS distortion on admission is better than STsegment measurements in predicting final infarct size and assessing the potential effect of thrombolytic therapy in anterior wall acute myocardial infarction. Am J Cardiol 1999;84: 530-534.

6. Birnbaum Y, Crigerda DA, Wagner GS, Strasberg B, MagerA, Gates K, et al. Prediction of extent and severity of left ventricular dysfunction in anterior acute myocardial infarction by the admission electrocardiogram. Am Heart J 2001a; 141: 915-924.

7. Birnbaum Y, Sclarovsky S, The grades of ischemia on the presenting electrocardiogram of patients with ST elevation acute myocardial infarction. Journal of Electrocardiology 2001b; 34 (4): 17 - 26.

8. Fernandez JR, Valle CMJ, Diaz MR, Pizarro G, Fernandez FL, Garcia RJM, et al. Terminal QRS distortion is an independent predictor of large area at risk and infarct size in patients with anterior stemi. $J$ Am Coll Cardiol 2015;65(10): 196.

9. Birnbaum Y, Drew BJ, The electrocardiogram in ST elevation acute myocardial infarction: correlation with coronary anatomy and prognosis. Postgraduate Medical Journal 2003; 79: 490-504.

10. Chowdhury R, Alam DS, Fakir II, Adnan SD, Naheed A ,Tasmin I, et al. The Bangladesh Risk of Acute Vascular Events (BRAVE) Study: objectives and design. Eur $J$ Epidemiol 2015; 30: 577-588.

11. Akanda MAK, Ali SY, Islam A, Rahman M M, ParveenA, Kabir M K, et al. Demographic Profile, Clinical Presentation \& Angiographic Findings in 637 patients with Coronary Heart Disease. Faridpur Medical College Journal 2011;6(2):82-85.

12. Yalcinkaya E, Yuksel U C, Celik M, Kabul H K, Barcin C, Gokoglan Y,et al. Relationship between neutrophil-tolymphocyte ratio and electrocardiographic ischemia grade in STEMI. Arquivos Brasileiros de Cardiologia 2015; 104(2): 112-119. 